\title{
A new approach based on principal ERPs and LDA to improve P300 mind spellers
}

This paper was downloaded from TechRxiv (https://www.techrxiv.org).

\section{LICENSE}

CC BY-NC-SA 4.0

SUBMISSION DATE / POSTED DATE

28-11-2021 / 06-12-2021

\section{CITATION}

Mobaien, Ali; Kheirandish, Negar; Boostani, Reza (2021): A new approach based on principal ERPs and LDA to improve P300 mind spellers. TechRxiv. Preprint. https://doi.org/10.36227/techrxiv.17088905.v1

DOI

10.36227/techrxiv.17088905.v1 


\section{A new approach based on principal ERPs and LDA to improve P300 mind spellers}

\author{
Ali Mobaien \\ Department of Electrical \\ and Computer Engineering \\ Shiraz University \\ Shiraz, Iran \\ Email: a.mobaien@shirazu.ac.ir
}

\author{
Negar Kheirandish \\ Department of Electrical \\ and Computer Engineering \\ Shiraz University \\ Shiraz, Iran \\ Email: negarkheirandish@yahoo.com
}

\author{
Reza Boostani \\ Department of Electrical \\ and Computer Engineering \\ Shiraz University \\ Shiraz, Iran \\ Email: boostani@shirazu.ac.ir
}

\begin{abstract}
Visual P300 mind speller is a brain-computer interface that allows an individual to type through his mind. For this goal, the subject sits in front of a screen full of characters, and when his desired one is highlighted, there will be a $\mathbf{P 3 0 0}$ response (a positive deflection nearly $300 \mathrm{~ms}$ after stimulus) in his brain signals. Due to the very low signal-tonoise (SNR) of the P300 in the background activities of the brain, detection of this component is challenging. Principal ERP reduction (pERP-RED) is a newly developed method that can effectively extract the underlying templates of event-related potentials (ERPs), by employing a three-step spatial filtering procedure. In this research, we investigate the performance of pERP-RED in conjunction with linear discriminant analysis (LDA) to classify P300 data. The proposed method is examined on a real P300 dataset and compared to the state-of-the-art LDA and support vector machines. The results demonstrate that the proposed method achieves higher classification accuracy in low SNRs and low numbers of training data.
\end{abstract}

Index Terms-Principal event-related potentials, $\mathbf{P 3 0 0}$, visual mind speller, brain-computer interface.

\section{INTRODUCTION}

Brain-computer interface (BCI) is a system which provides a communication channel between mind and computer, by translating brain signals [1], [2]. One of the most convenient ways to record brain activities is electroencephalography (EEG). EEG is non-invasive, cheap, and accessible compared to other methods [3]. One type of EEG-based BCI system is the P300 speller which allows an individual to type characters through his mind [4]. P300 is a kind of event-related potential (ERP) that can be elicited by an oddball setting [5]. In such settings, the subjects are exposed to a series of frequent (non-target) and rare (target) visual or auditory stimuli. Research findings indicate that, when a subject is exposed to an infrequent (target) stimulus, about $300 \mathrm{~ms}$ post-stimulus, there is a positive deflection in his brain response. This response can be used as a cue to control a mind speller device. An example of the P300 speller paradigm can be a matrix of alphabets suggested by Farwell et al. [4]. In this setup, the system intensifies each row or column of the matrix in a random fashion. When the row or column corresponding to the interested character is intensified, there will be a P300 signal in the subject's EEG, and the character can be identified (by the intersection of the row and column of interest).
Due to the low signal-to-noise (SNR) of P300 in the background EEG, P300 detection (i.e. target trial) is challenging and usually needs several trials to detect this component with acceptable confidence. Trial averaging of post stimuli signals is a usual method to increase SNR level. By assuming the independence between the activities of background EEG (nearly random behavior) and a near to deterministic morphology of P300 regarding stimuli onset, trial averaging can cancel out the background noise and increase SNR [6]. The more trials for averaging, the more P300 becomes clear. However, collecting many trials is time-consuming and leads to low bit rates, not applicable for practical purposes. Also, in long sessions, the data's quality is decreased (P300 level decreases, and the noise level increases). This is due to brain fatigue and getting used to periodic stimuli. Brain fatigue reduces the P300 amplitude and increases its latency. Hence, new and applicable signal processing techniques to more accurately detect and estimate the P300 are very useful.

P300 detection or classification is an important issue in the field of biomedical signal processing, hence, the domain is fairly mature. There are a vast number of studies that have addressed this problem, and they are mostly focused on either designing a better paradigm to evoke the P300 [7] or signal processing stage [8]. The scope of this paper is the latter. According to [8], the most favorite P300 classifiers are based on linear discriminant analysis (LDA) [9] and support vector machines (SVM) [10] due to achieving very good results.

It is well-known that the observed ERP signals are the summation of several waveforms. For instance, P300 consists of two subcomponents namely $\mathrm{p} 3 \mathrm{a}$ and $\mathrm{p} 3 \mathrm{~b}$ where $\mathrm{p} 3 \mathrm{a}$ arrives first and is mostly related to the attention, distributed frontally on the scalp, and p3b is mostly related to memory update, originated from temporal-parietal scalp regions [5]. In a recent work by Campos et al. (2020), a new approach toward studying ERPs has been developed [11]. The underlying idea of the proposed method is that any ERP signal can be described by a set of basic templates called principal ERPs (pERPs). These basis waveforms are extracted using a three-step spatial filtering method called pERP-reduction (pERP-RED). PERPRED has performed very successfully to analyze different ERPs. 


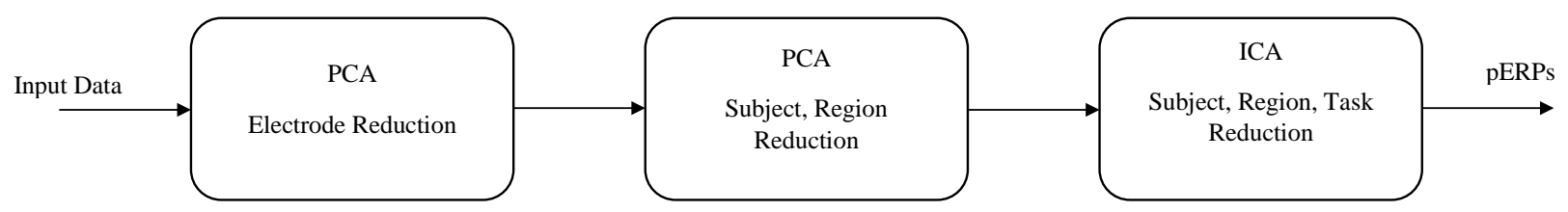

Fig. 1. The structure of pERP-RED method for pERPs extraction. This method use three steps of spatial filtering over EEG data of different subjects during different tasks, captured from several electrodes.

Contribution: To the author's knowledge, the pERP-RED algorithm has not been investigated for P300 classification up to now. Hence, in this paper, we develop a rather simple method based on pERPs and LDA (pERP-LDA), which shows to be very effective for emerging $\mathrm{P} 300$. The proposed method is examined on a real P300 dataset from BCI competition III [12] and compared to LDA and SVM. The results demonstrate that the proposed method achieves a higher classification accuracy in low SNRs. This leads to higher bit rates which are very important to the real-life applicability of mind spellers. Furthermore, the purposed pERP-LDA shows to have a better performance in the case of small training sets, which means a faster calibration of P300 speller systems.

\section{METHOD}

\section{A. Overview of PERP-RED Algorithm}

The basic assumption of the pERP-RED method [11] is that any observed ERP signal at any electrode, for any subject, and any task can be described by a linear combination of underlying ERP waveforms (i.e. pERPs). To extract the pERPs, three levels of spatial filtering are employed (Fig.1). Let $X_{v}^{i} \in \mathbb{R}^{T \times E_{i}}$ be a post-stimulus data matrix for $i$ 'th subject $(i=1,2, \ldots, N)$ and $v$ 'th task $(v=1,2, \ldots, V)$, where $T$ is the number of recorded samples in time and $E_{i}$ is the number of recorded channels for $i$ 'th subject. The data are scaled and biased to have a zero mean and unit variance in each channel. In the first step, the data matrices of each subject are concatenated in the columns (leading to $(T \times V) \times E_{i}$ matrices). Then an electrode reduction step is done through principal component analysis (PCA) to eliminate highly correlated channels. This leads to a smaller set of uncorrelated bases called principal regions. The number of these regions $\left(R_{i}\right)$ could be different for each subject, and it depends on a pre-specified amount of variations to preserve (by default the threshold is $80 \%$; larger thresholds mean preserving more data, but at the cost of keeping more noise as well). It is obvious that $R_{i} \leqslant E_{i}$. The second step is dedicated to subject-region reduction. For this, the resulting matrices from the first step and all the subjects are concatenated in the rows leading to a big matrix of size $(T \times V) \times \sum_{i=1}^{N} R_{i}$. Each task-region ERP record is normalized to unit variance. Then again, PCA is used to generate $N_{R}$ principal subject-regions based on a pre-specified amount of variance (by default the threshold is $80 \%)$. This results in a $(T \times V) \times N_{R}$ matrix. At last, the latter mentioned matrix is reshaped to a matrix of size $T \times\left(V \times N_{R}\right)$, then independent component analysis (ICA) is used to extract $P$ principle ERPs. $P$ could be specified based on a priori information about ERP structure (e.g. $P=2$ for $\mathrm{P} 300$, as it consists of two main subcomponents [5]), or it could be found by excluding a small set of data from the training set and using it to find the number of required pERPs [11].

Consider $\Phi=\left[\phi_{1}, \phi_{2}, \ldots, \phi_{P}\right]$ as a matrix of extracted pERPs. Now each observed data matrix $X_{v}^{i}$ can be expressed by a linear combination of these pERPs through the following regression (note that the data must be biased to have a zero mean in each channel),

$$
\hat{X}_{v}^{i}=\Phi\left(\Phi^{t r} \Phi\right)^{-1} \Phi^{t r} X_{v}^{i}
$$

where $\{.\}^{t r}$ denotes transpose operator. This can also be seen as a column-wise regression of $X_{v}^{i}$ on pERPs. One may consider $\hat{X}_{v}^{i}$ as a filtered version of $X_{v}^{i}$ through $F_{\text {erp }}=$ $\Phi\left(\Phi^{t r} \Phi\right)^{-1} \Phi^{t r}$.

A ready-to-use package of the pERP-RED algorithm can be found at www.github.com/emjcampos/perpred. This package is provided in $\mathrm{R}$ language [13].

\section{B. Proposed pERP-LDA}

In the studies of $\mathrm{P} 300$ classification, it is common practice to pass the data through a filter (temporal, spatial, or spatiotemporal) to enhance $\mathrm{P} 300$, and then, feed it to a classier to find the final label (target or non-target). Our proposed method also consists of two main successive blocks, a filter pERPs $\left(F_{\text {erp }}\right)$ to enhance the P300, and an LDA classifier to find the final label.

In the training phase, first, the pERPs are found through the pERP-RED algorithm using the train data, then, the same data are projected on pERPs through $F_{\text {erp }}$. At last, the filtered data are used to train the LDA classier. In the testing phase, first, the test data are passed through $F_{\text {erp }}$ to enhance the P300, then, the filtered data are fed to the LDA classifier to find the final label.

\section{Method Evaluation}

\section{A. P300 Data}

The pERP-LDA method is examined using a real visual P300 speller dataset from BCI competition III [12]. This dataset consists of EEG recordings from two subjects (A and B) while trying to spell 185 different characters. For this, a matrix of characters was shown to the users while they were asked to focus on the desired one. All rows and columns of this matrix were successively and randomly intensified, 


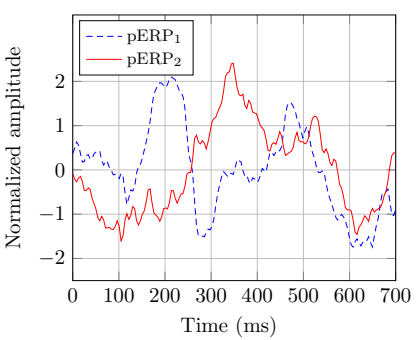

(a)

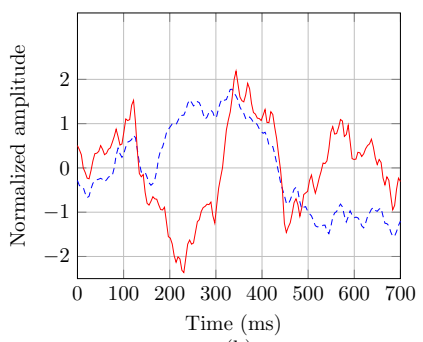

(b)

Fig. 2. The extracted pERPs through pERP-RED. (a) Subject A. (b) Subject B

which two of them (one row and one column) contained the desired character. This procedure has been repeated 15 times to collect more data for each letter. Data was captured from 64 channels using 10-20 standard, filtered between 0.1 to $60 \mathrm{~Hz}$, and sampled at $240 \mathrm{~Hz}$.

To perform further experiments on the dataset, a data segmentation step is required to extract the target and nontarget trials from the post stimuli EEG recordings. For each character, the data sequence is split into twelve $700 \mathrm{~ms}$ blocks (corresponding to twelve rows and columns) where two of them are target trials. This leads to $185 \times 10$ non-target and $185 \times 2$ target trials for each subject. Each of these blocks contains 15 subsequences corresponding to 15 repetitions of each row or column flash in a chronological manner. The data blocks are captured from four channels $\left(C_{z}, \mathrm{PO}_{7}, \mathrm{~F}_{z}\right.$ and $\left.\mathrm{P}_{4}\right)$ which mostly reflect the P300 activity [8].

\section{B. Evaluation Scheme and Results}

The proposed pERP-LDA is compared with LDA and SVM in terms of classification accuracy. To this aim, the competing methods are evaluated on two equal-size groups of randomly chosen target and non-target trials, through 10-fold crossvalidation. The methods are also evaluated in different SNRs and different amounts of training data. To increase the SNR, the trials are averaged over the first $k$ repetitions.

To enhance the $\mathrm{P} 300$ and reduce the noise, the data are bandpass filtered, by a 0.1 to $15 \mathrm{~Hz}$ [14] Butterworth filter of order 4. The filtered data are fed to LDA and SVM classifiers, however, the raw data are fed to pERP-LDA as it benefits from a built-in noise cancelation procedure. The number of pERPs is set to 2 (as there are mainly two subcomponents in the P300), and the threshold is set to $80 \%$ for the electrode reduction step.

In the following, the results in Fig. 2 to Fig. 5 are achieved using two-channel data $\left(\mathrm{C}_{z}\right.$ and $\left.\mathrm{PO}_{7}\right)$. Fig. 2 indicates the pERPs extracted for subject A (Fig. 2a) and subject B (Fig. $2 b)$. For both subjects, it can be seen that $\mathrm{pERP}_{1}$ picks first about $200 \mathrm{~ms}$, and $\mathrm{pERP}_{2}$ picks later about $350 \mathrm{~ms}$. Hence, these components could be closely related to $\mathrm{p} 3 \mathrm{a}$ and $\mathrm{p} 3 \mathrm{~b}$. Figs. 3 and 4 show the performance of pERP-LDA, LDA, and SVM in different SNRs (i.e. different $k$ values) using two training sets with sizes of 20 and 370 . For both subjects, the proposed method provides higher accuracy almost in all

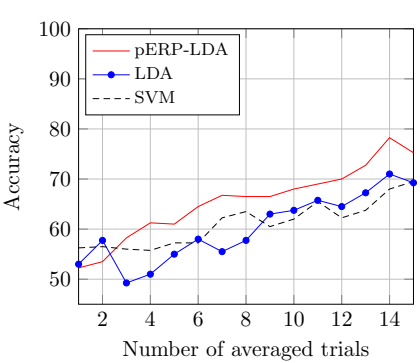

(a)

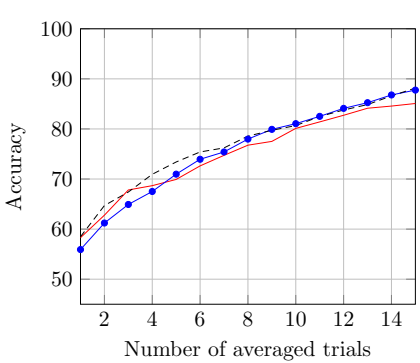

(b)
Fig. 3. Subject A, the performance of pERP-LDA, LDA, and SVM in different SNRs. The size of the training set is (a) 20; (b) 370 .

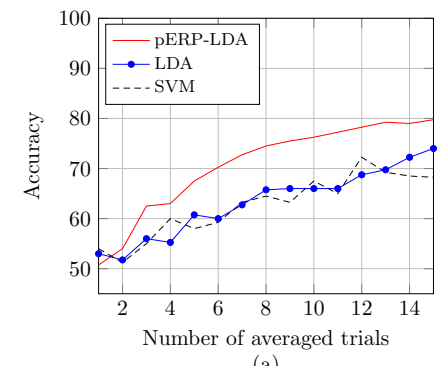

(a)

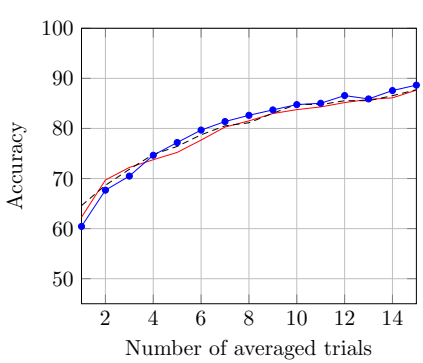

(b)
Fig. 4. Subject B, the performance of pERP-LDA, LDA, and SVM in different SNRs. The size of the training set is (a) 20; (b) 370 .

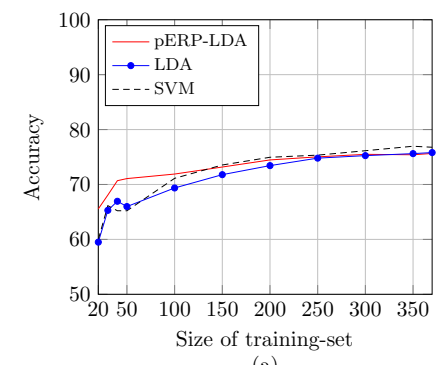

(a)

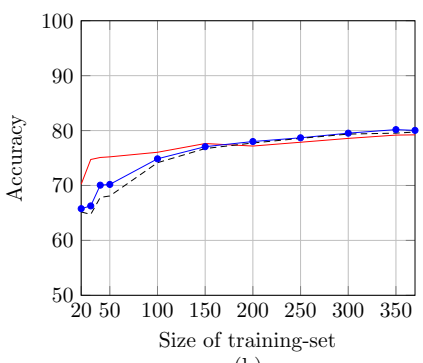

(b)
Fig. 5. The performance of pERP-LDA, LDA, and SVM in different amounts of training data. (a) Subject A. (b) Subject B.

SNR levels over the small training set and performs as well as LDA and SVM over the large training set. The average performances of competing methods over different sizes of the training set are shown in Fig. 5. It can be seen that, for both subjects, pERP-LDA outperforms the other two methods in small amounts of training data. This can be more clearly seen for training sets of size 150 or less. For instance, the LDA and SVM need 100 training data to achieve the same performance as pERP-LDA with only 30 training data (Fig. 5b). By increasing the size of the training set, the performance gaps between pERP-LDA and competing methods decrease which is due to better estimation of unknown model parameters of LDA and SVM classifiers. Figs. 6 and 7 indicate the average performance of different methods using different numbers of EEG channels. Again, the same results as before are seen. For both subjects and all different channel setups, the proposed method outperforms LDA and SVM in small amounts of 


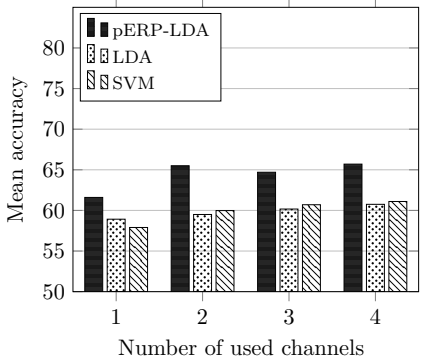

(a)

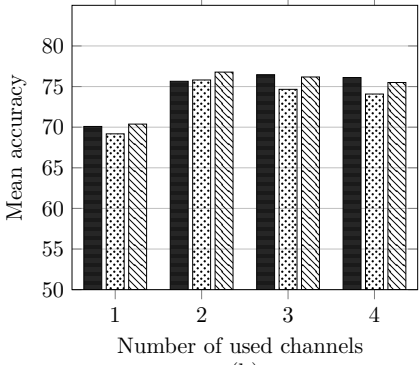

(b)
Fig. 6. The performance of different methods using different numbers of EEG channels for subject A. (a) Training-set size is 20. (b) Training-set size is 370 .

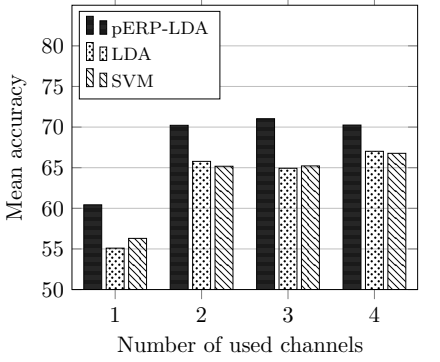

(a)

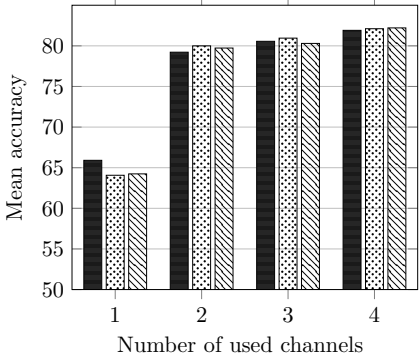

(b)
Fig. 7. The performance of different methods using different numbers of EEG channels for subject B. (a) Training-set size is 20. (b) Training-set size is 370 .

data (Figs. 6a and 7a), however, for large training sets, the performance of all methods are rather close (Figs. 6b and 7b).

\section{Discussion AND CONCLUSION}

In this study, we proposed a new approach called pERPLDA for P300 classification in mind speller paradigms. This method is based on a recent research regarding ERP analysis (pERP-RED [11]) and LDA classifier. PERP-RED extracts the waveforms which are believed to represent the underlying activities of ERPs. P300 is a well studied ERP component and it consists of two main subcomponents named p3a and p3b [5]. In Fig. 2, the extracted pERPs from real P300 data are closely related to these subcomponents which verifies the effectiveness of the pERP-RED method. When the principal ERPs are found, the P300 trials of any type (i.e. target or nontarget), and for any electrode could be represented by them. This leads to effective noise reduction and P300 enhancement.

The results suggest that pERP-LDA provides higher classification accuracies for small training sets and low SNR situations, compared to traditional classifiers (LDA and SVM). This is very promising as many P300-based mind spellers require large training datasets to have a good performance, making their calibration time very long. Also, good performance in a low number of repetitions (i.e. low SNRs) means a higher bit rate of the speller. Putting these two together, approaches the P300 speller to an applicable system in real-life situations. In future studies, the effectiveness of the proposed method should be investigated over different datasets and different ERPs. Also, the combination of pERP-RED with deep structures [1] may lead to a universal ready-to-use mind speller which is of great interest.

\section{REFERENCES}

[1] M. Dehghani, A. Mobaien, and R. Boostani, "A deep neural networkbased transfer learning to enhance the performance and learning speed of BCI systems," Brain-Computer Interfaces, vol. 8, no. 1-2, pp. 14-25, 2021.

[2] A. Mobaien and R. Boostani, "ACSP: Adaptive CSP filter for BCI applications," in 2016 24th Iranian Conference on Electrical Engineering (ICEE). IEEE, 2016, pp. 466-471.

[3] D. L. Schomer and F. L. Da Silva, Niedermeyer's Electroencephalography: Basic Principles, Clinical Applications, and Related Fields. Lippincott Williams \& Wilkins, 2012.

[4] L. A. Farwell and E. Donchin, "Talking off the top of your head: Toward a mental prosthesis utilizing event-related brain potentials," Electroen. Clin. Neuro., vol. 70, no. 6, pp. 510-523, 1988.

[5] T. W. Picton, "The P300 wave of the human event-related potential," $J$. Clin. Neurophysiol., vol. 9, no. 4, pp. 456-479, 1992.

[6] H. Parvar, L. Sculthorpe-Petley, J. Satel, R. Boshra, R. C. D'Arcy, and T. P. Trappenberg, "Detection of event-related potentials in individual subjects using support vector machines," Brain Informatics, vol. 2, no. 1, pp. 1-12, 2015.

[7] G. Townsend, B. K. LaPallo, C. B. Boulay, D. J. Krusienski, G. Frye, C. Hauser, N. E. Schwartz, T. M. Vaughan, J. R. Wolpaw, and E. W. Sellers, "A novel P300-based brain-computer interface stimulus presentation paradigm: Moving beyond rows and columns," Clin. Neurophysiol., vol. 121, no. 7, pp. 1109-1120, 2010.

[8] J. T. Philip and S. T. George, "Visual P300 mind-speller brain-computer interfaces: A walk through the recent developments with special focus on classification algorithms," Clin. EEG Neurosci., 2019.

[9] J. Qu, F. Wang, Z. Xia, T. Yu, J. Xiao, Z. Yu, Z. Gu, and Y. Li, "A novel three-dimensional P300 speller based on stereo visual stimuli," IEEE Trans. Human-Mach. Syst., vol. 48, no. 4, pp. 392-399, 2018.

[10] M. Kaper, P. Meinicke, U. Grossekathoefer, T. Lingner, and H. Ritter, "BCI competition 2003-data set IIb: Support vector machines for the P300 speller paradigm," IEEE Trans. Biomed. Eng., vol. 51, no. 6, pp. 1073-1076, 2004.

[11] E. Campos, C. Hazlett, P. Tan, H. Truong, S. Loo, C. DiStefano, S. Jeste, and D. Şentürk, "Principle ERP reduction and analysis: Estimating and using principle ERP waveforms underlying ERPs across tasks, subjects and electrodes," NeuroImage, vol. 212, p. 116630, 2020.

[12] J. R. Wolpaw, G. Schalk, and D. Krusienski. (2005) BCI competition III webpage. [Online]. Available: http://www.bbci.de/competition/iii/

[13] E. Campos, C. Hazlett, and D. Senturk, pERPred: Principle 'ERP' Reduction, 2021, r package version 0.0.0.9000. [Online]. Available: https://github.com/emjcampos/pERPred

[14] L. Bougrain, C. Saavedra, and R. Ranta, "Finally, what is the best filter for P300 detection?" in TOBI Workshop lll-Tools for Brain-Computer Interaction-2012, 2012. 